\title{
Quali alterazioni dell'esame urine possono preludere ad un interessamento renale della malattia di Fabry
}

Giornale di Tecniche Nefrologiche e Dialitiche 2019, Vol. 31(2) 137-139

(C) The Author(s) 2019

Article reuse guidelines:

sagepub.com/journals-permissions DOI: $10.1177 / 0394936219851960$ journals.sagepub.com/home/gtn

(S)AGE

\section{Sandro Feriozzi e Mario Mangeri}

\begin{abstract}
Urinary markers in Anderson-Fabry nephropathy

The markers of renal involvement in Anderson-Fabry disease are defects of urine concentration, presence of cells with lipid cytoplasmatic inclusions (mulberry bodies) and podocyturia. The loss of urine concentrating capacity is not easy to detect and the search for cellular inclusions is a complex technique. Moreover, none of the markers has any clear correlation with the stage of the disease. The occurrence of podocytes in the urine (podocyturia) correlates with both renal involvement and clinical outcome; therefore, podocyturia seems to be a promising early indicator of nephropathy. However, a common agreement on the laboratory assay used to measure is still needed.
\end{abstract}

\section{Keywords}

Tubular defects, Mulberry bodies, Podocyturia

\section{Introduzione}

Sin dalle prime segnalazioni della malattia di Anderson-Fabry $(\mathrm{AF})$ in letteratura, i reperti urinari sono stati oggetto di studio da parte dei ricercatori. Le peculiarità urinarie nella malattia di AF derivano dalle caratteristiche intracellulari dovute alla deposizione di lipidi complessi (Gb3) non degradati nelle strutture renali. ${ }^{1}$ Tale deposizione avviene sia nell'endotelio dei capillari e delle arterie sia nell'epitelio del glomerulo (podociti) e dei tubuli. La deposizione nei vasi determina stimolazione delle cellule muscolari lisce della parete e attivazione di citochine pro-ossidanti ed infiammatorie, ma non ha un diretto corrispettivo nelle urine. ${ }^{2}$ La deposizione nelle cellule tubulari avviene soprattutto nei citoplasmi delle cellule distali mentre a livello podocitario è nota la frequente deposizione associata a comparsa di proteinuria. Questi quadri clinici-laboratoristici sono classificabili secondo le seguenti categorie di marker: alterazione della concentrazione urinaria, corpi cellulari inclusi nelle urine, presenza di podocituria.

Questi reperti sono, almeno teoricamente, facilmente accessibili e le indagini facilmente ripetibili, per cui molti ricercatori hanno focalizzato la loro attenzione su di essi. In particolare si è cercato di capire se possano essere degli indicatori precoci di danno renale. Quest'ultimo punto ha un riflesso molto importante stante l'ormai acquisito concetto che la terapia nella $\mathrm{AF}$ è tanto più efficace quanto più l'inizio è precoce. ${ }^{3}$

\section{Difetti tubulari}

La deposizione di $\mathrm{Gb} 3$ avviene soprattutto nelle cellule dei tubuli distali, dove fisiologicamente è stata dimostrata la presenza di lipidi complessi. ${ }^{4}$ Pertanto in corso di AF la cellula del tubulo distale è la sede principale della deposizione. Tale differenza è facilmente apprezzabile nelle biopsie renali eseguite di routine nei pazienti affetti da $\mathrm{AF}$ dove le cellule con le vacuolizzazioni citoplasmatiche sono quelle del tubulo distale (figura 1). Questo determina che, dal punto di vista clinico, il difetto maggiore sia una riduzione dei processi di riassorbimento della pre-urina e

UOC Nefrologia e Dialisi, ospedale Belcolle, Viterbo

\section{Corrispondenza:}

Sandro Feriozzi, Nefrologia e Dialisi, Ospedale Belcolle, Strada Sammartinese snc, 01100 Viterbo, Italy.

E-mail: sandro.feriozzzi@asl.vt.it 


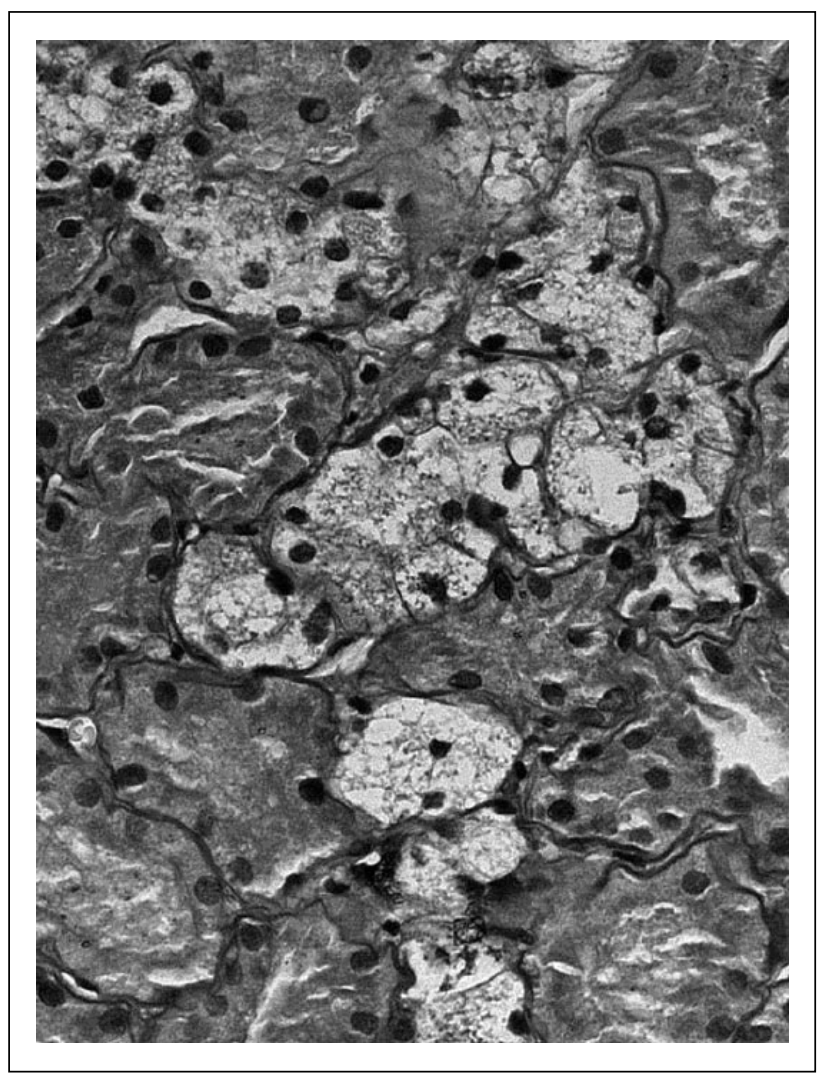

Figura I. Microscopia ottica di biopsia renale di paziente con malattia di Anderson-Fabry. Si possono osservare cellule del tubulo distale con numerose vacuolizzazioni citoplasmatiche mentre le cellule prossimali (con orletto a spazzola) sono prive di vacuolizzazioni (40X PAS).

quindi una ridotta capacità di concentrare le urine associata a poliuria con nicturia. E' stata descritta anche una ridotta capacità all'ammoniogenesi ed un'acidosi tubulare distale. ${ }^{5}$

La controparte clinica dei difetti di concentrazione urinaria non è facilmente diagnosticabile in quanto richiede delle indagini di laboratorio particolari e non di ampia diffusione nei reparti di nefrologia. Pertanto, seppur interessanti da un punto di vista speculativo, i difetti tubulari non si prestano per una diagnosi precoce e per un monitoraggio della terapia nel tempo.

\section{Inclusioni cellulari}

L'eliminazione con le urine dei lipidi contenuti nelle cellule del tubulo è evento frequente nella malattia di AF. Le cellule esaminate con la luce polarizzata assumono la conformazione detta a croce di malta m maiuscola (figura 2) o mulberry bodies. Tale reperto non è specifico solo della AF ma può essere visto in corso di altre nefropatie associate a proteinuria grave e lipiduria. Queste figure cellulari sono costituite da cellule tubulari con all'interno le deposizioni di $\mathrm{Gb} 3$ e quindi desquamate e eliminate con le urine.

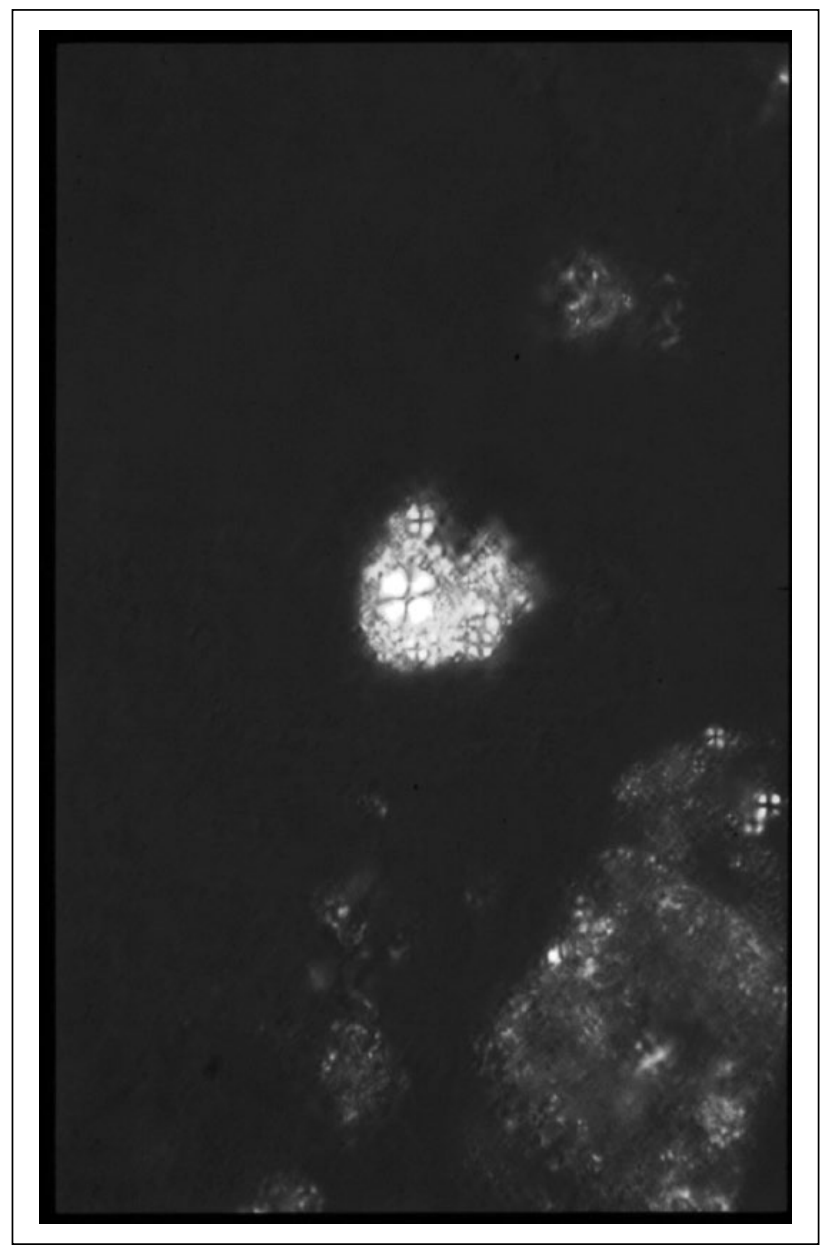

Figura 2. Microscopia ottica con luce polarizzata. Si osserva la birifrangenza dei lipidi contenuti nelle cellule eliminate con le urine con aspetto tipico detto a croce di Malta (X40).

Selvarajan ${ }^{6}$ ha dimostrato, con diverse colorazioni di istochimica e con anticorpi, la presenza del Gb3 nel citoplasma di tali cellule e valutato tale reperto come indice diagnostico in 35 soggetti con malattia di AF messi a confronto con urine di soggetti con altre nefropatie. Il riscontro di queste inclusioni cellulari aveva un'alta sensibilità diagnostica in ambo i sessi ma richiedeva una procedura di preparazione non semplice del sedimento urinario, nonché un certa esperienza da parte del lettore. Non vi era tuttavia una correlazione con lo stato clinico della nefropatia e la loro presenza è indicativa di AF solo nell'ambito di quadri clinici tipici.

\section{Podocituria}

I podociti sono cellule altamente differenziate, con scarsa o nulla capacità di reduplicarsi, che sintetizzano le proteine che formano la barriera di filtrazione (slit-diaphram) glomerulare e che regolano la permeabilità della membrana basale. Negli ultimi anni si è visto che i podociti possono essere compromessi in processi patologici come alterazioni genetiche delle proprie proteine strutturali, lesioni dovute a 
mediatori dei processi infiammatori, e danni indotti dallo stress emodinamico. In queste situazioni, il podocita subisce l'azione di processi quali l'attività di proteinchinasi, lo stress ossidativo,e il danno mitocondriale. Questi processi provocano alterazioni funzionali, un danno al citoscheletro e infine il podocita si stacca dalla membrana, muore e può essere ritrovato nelle urine. ${ }^{7}$

Il riconoscimento dei podociti nelle urine viene fatto attraverso diverse metodiche: immunofluorescenza con riconoscimento di proteine, RNA polimerasi, cromatografia liquida con tandem mass. ${ }^{8}$ Queste diverse tecniche non danno risultati completamente sovrapponibili e quindi generano una difficoltà nel valutare il ruolo della podocituria nella malattia di AF.

La podocituria è stata descritta in alcune patologie renali come la nefropatia diabetica, la nefropatia IgA e la pre-eclampsia. Pertanto si deve precisare che non è specifica dei soli pazienti con malattia di $\mathrm{AF}^{7,8}$

Nella malattia di AF vi sono numerose segnalazioni sulla presenza e sul ruolo della podocituria. In particolare, Trimarchi, ${ }^{9}$ utilizzando anticorpi verso le proteine strutturali dei podociti, ha descritto la presenza di un'elevata podocituria nelle urine dei pazienti con AF, inoltre la podocituria precede la comparsa della stessa proteinuria. Il danno dei podociti nella malattia di AF si ritiene sia dovuto alla deposizione di Gb3 nel citoplasma con attivazione di meccanismi patologici come la sintesi di citochine infiammatorie e attivazione dello stress ossidativo. Trimarchi analizza anche la presenza di alcune proteine marker dei podociti urinari e descrive un possibile andamento di queste proteine. Nel tempo, nei podociti si avrebbe una riduzione della podocalixina, proteina di ancoraggio alla membrana basale, e sarebbe possibile repertare la sola sinaptopodina, proteina del citoscheletro, che diventa il marker rintracciabile della podocituria. L'andamento nel tempo della podocituria nella malattia di AF potrebbe essere caratterizzato da un aumento nelle fasi precoci della nefropatia, successivamente, con il danno glomerulare, il numero dei podociti si riduce e quindi la stessa podocituria sarà ridotta. Alternativamente si suggerisce che le diverse espressioni delle proteine marker nei podociti possano essere dovute a modifiche nel tempo dei fenotipi. Un elemento estremamente importante da considerare è che la podocituria sembra correlare al valore del LysoGb3 plasmatico ${ }^{8}$ che è marker diagnostico, ma anche un valido surrogato per valutare la gravità della malattia.

La podocituria nella AF quindi potrebbe avere un ruolo importante nel segnalare il danno renale sin dalle primissime fasi e quindi potrebbe essere un indicatore per iniziare precocemente la terapia. Resta tuttavia un limite significativo rappresentato dalle tecniche di laboratorio che sono sofisticate e non completamente sovrapponibili nei risultati, e quindi non è ancora possibile standardizzare i risultati. ${ }^{10}$

\section{Dichiarazione di assenza di conflitto di interessi}

Gli Autori dichiarano di non avere conflitti di interessi.

\section{Finanziamenti}

Gli Autori dichiarano di non aver ricevuto finanziamenti specifici da qualsiasi ente nei settori pubblico, privato o senza fini di lucro.

\section{Bibliografia}

1. Desnick RJ, Brady R, Barranger J, et al. Fabry disease, an underrecognized multisystemic disorder: expert recommendations for diagnosis,management, and enzyme replacement therapy. Ann Intern Med 2003; 138:338-346.

2. Rombach SM, Twickler ThB, Aerts JM, et al. Vasculopathy in patients with Fabry disease: current controversies and research directions. Mole Genet Metab 2010; 99:99-108.

3. Germain DP, Elliot PM, Falissant B, et al. The effect of enzymatic therapy on clinical outcomes in male patients with Fabry disease: a systematic literature review by a European panel of experts. Mol Genet Metab Dis 2019; 19:100454.

4. Faraggiana T, Crescenzi A and Marinozzi V. Presence of an alfa-galactopid on the cell surface of endothelial cells of human kidney. Biochem J 1989; 21:235-240.

5. Moran V, Obrador GT and Thadhami R. Fabry kidney disease. Saudi J Kidney Organ Transplantation 2003; 14:367-377.

6. Selvarajah M, Nicholls K, Hewitson TD and Becker GJ. Target urine microscopy in Anderson-fabry disease: a cheap sensitive and specific technique. Nephrol Dial Transplant 2011; 26:3195-3262.

7. Trimarchi H and Coppo R. Podocytopathy in mesangial proliferative immunoglobulin A nephropathy: new insights into the mechanisms of damage and progression. Nephrol Dial Transplant 2009; doi: 10.1093/ndt/gfy413. [Epub ahead of print]

8. Martineau T, Boutin M, Côté AM, Maranda B, Bichet DG and Auray-Blais C. Tandem mass spectrometry analysis of urinary podocalyxin and podocin in the investigation of podocyturia in women with preeclampsia and Fabry disease patients.Clin Chim Acta 2019; 495:67-75.

9. Trimarchi H, Canzonieri R, Costales Collaguazo C, et al. Early decrease in the podocalyxin to synaptopodin ratio in urinary Fabry podocytes. Clin Kidney J 2019; 12:53-60.

10. Sanchez-Nino MD, Perez-Gomes MV, Valino-Rivas L, et al. Podocyturia: why it may have added value in rare diseases. Clin Kidney J 2018; 12(1):49-52. 